\title{
Processing Centroids of Smearing Star Image of Star Sensor
}

\author{
Yufu Liao, ${ }^{1,2}$ Enhai Liu, ${ }^{1}$ Jianyong Zhong, ${ }^{1}$ and Hui Zhang ${ }^{1}$ \\ ${ }^{1}$ Institute of Optics and Electronics, Chinese Academy of Science, Mailbox 350, Chengdu, Sichuan 610200, China \\ ${ }^{2}$ University of Chinese Academy of Science, Beijing 100191, China \\ Correspondence should be addressed to Yufu Liao; sduliaoyufu@163.com
}

Received 4 November 2013; Revised 10 March 2014; Accepted 10 March 2014; Published 14 April 2014

Academic Editor: Xin Wang

Copyright (C) 2014 Yufu Liao et al. This is an open access article distributed under the Creative Commons Attribution License, which permits unrestricted use, distribution, and reproduction in any medium, provided the original work is properly cited.

\begin{abstract}
A novel method was presented for increasing the accuracy of subpixel centroid estimation for smearing star image. Model of the smearing trajectory of smearing star was built. It helped to study the analytical form of the errors, caused by image smearing, for centroid estimation. In the algorithm, the errors were estimated with accuracy and used to revise the centroid processed by CoM (centre of mass). Simulations have been run to study the effect of angular rates, integration time, and actual position of star on the accuracy of centroid estimation. Results were presented which suggested that the proposed algorithm had a precision better than $1 / 10$ of a pixel when the angular rate was up to $3.0 \mathrm{deg} / \mathrm{s}$.
\end{abstract}

\section{Introduction}

A star sensor is a device which outputs pose for its carrier by measuring the directions of stars in the FOV (field of view). Those directions are compared with star directions stored in star catalog. Then pose of the carrier can be determined. Being one of the most accurate means for pose determination with accuracies down to arc seconds $[1,2]$, star sensor has been used in a variety of spacecrafts and experiments with its increasing accuracies.

In static condition angular rates of the star sensor are low, usually less than $0.3 \mathrm{deg} / \mathrm{s}$. Intensity of a star imaged is often considered following 2D Gaussian distribution in engineering. Adopting interpolation or Gaussian centroid, the processed centroid can be better than $1 / 10$ of a pixel [3]. Contrarily, angular rates are high in dynamic condition. Imaged stars are nonideal that their gray intensity does not follow 2D Gaussian distribution. Accuracy of the processed centroid might be worse than several pixels.

Many works have been done on processing centroid for smeared star. Xiaojuan and Xinlong [4] have described how the three-axis angular rates affect the centre determination of star spots. Weina et al. [5] have described a method to restore the smeared star image before processing centroid, while only the smear caused by velocities of cross-sight direction was analyzed. Image deconvolution could increase the ability to detect stars for a sensor while stars in the deconvolved image present a nonusual aspect (with several peaks) [6]. A gyrocontrol platform might be designed to eliminate the effect caused by angular movement of the spacecraft. But this scheme resulted in increased size, weight, and complexity of the star sensor. Adjusting the integration time to the variation of the angular rates can weaken the effect on processing centroid, while smear is inevitable and errors caused by the smearing still cannot be neglected. In this paper, it is hoped that the question will be resolved with a better way.

To overcome the difficulties arisen from smeared star image, a novel and effective method was presented in this paper, aiming at obtaining better centroid. In our case, the smearing trajectory of star was deduced firstly. Then, within the allowed precision it was approximated by Taylor's series. After that, analytical expressions of errors, resulting from image smearing when CoM was applied, were deduced. The errors are related to the unknown actual position. Combining the relation between centroid by CoM and the actual position, the errors could be expressed by centroid processed by CoM. Lastly, a novel and efficient algorithm was proposed to obtain better centroid for smearing star image.

This paper consists of eight sections. Section 1 makes a brief introduction of the background and significance of the study. After this introduction, the mechanism of operation of a typical star sensor and its imaging geometrical model are outlined. Section 3 introduces the trajectory of smearing star. Section 4 further studies the mathematical model of the 
smearing star and its trajectory is approximated by polynomial from engineering perspective. In Section 5, errors of the processed centroid by CoM for smeared star are studied. Main steps of our method are drawn in Section 6. Simulations are constructed to verify the feasibility and efficiency of our method in Section 7. Lastly, conclusions are drawn in Section 8 .

\section{Performance of Star Sensor}

A star sensor usually consists of sensor system and dataprocessing system. It captures star images with its electronic camera. Afterward, a series of sequential implementations, including star image pretreatment, processing centroid, identification, and star pattern recognition are constructed. After that, it outputs the pose of the carrier.

The pose of carrier is often parameterized by a $3 \times 3$ direct cosine matrix $A$ which satisfies the following equation:

$$
\widehat{U}=A \widehat{V}
$$

where $\widehat{U}$ and $\widehat{V}$ denote the unit vectors in the body-fixed frame and the inertial based coordinates system, respectively. For pose determination, the star sensor observes directional vectors $\widehat{U}$ of stars, while $\widehat{U}$ are derived from processed centroids. And then it obtains the reference vectors $\widehat{V}$ from star catalog by identifying star constellations. However, centroids without high precision would result in a worse $\widehat{U}$ and decrease the success rate of identifying star constellations. It is suggested that the much more accurate centroids might lay a solid foundation for star sensor to output more accurate pose of the spacecraft [1].

The imaging geometrical model of star sensor is drawn in Figure 1. All the analysis after this section is based on the coordinate system shown in it. $\omega_{x}, \omega_{y}$, and $\omega_{z}$ are the angular rates around $x$-axis, $y$-axis, and $z$-axis, respectively. $f$ is the focal length and $(x, y)$ denotes the position of a star on the focal plane.

\section{Smearing Trajectory}

In static condition, a star sensor obtains dispersive star spot of 3 by 3 to 7 by 7 pixels by defocusing method. Maximum angular rate that a star sensor can endure in order to output pose with required precision might be estimated briefly [7]. However, errors caused by image smearing cannot be ignored when the angular rate is two or three times the maximum one.

Dynamic variation of coordinate $x$ is related to $\omega_{y}$ and $\omega_{z}$ (Figure 1). $f \omega_{y}$ and $-y(t) \omega_{z}$ are the two components of the $x$-axis line velocity. The synthesized line velocity about time $t$ can be described as follows:

$$
x^{\prime}(t)=f \omega_{y}-y(t) \omega_{z}
$$

In the same way, another differential expression about time $t$ could also be obtained:

$$
y^{\prime}(t)=-f \omega_{x}+x(t) \omega_{z}
$$

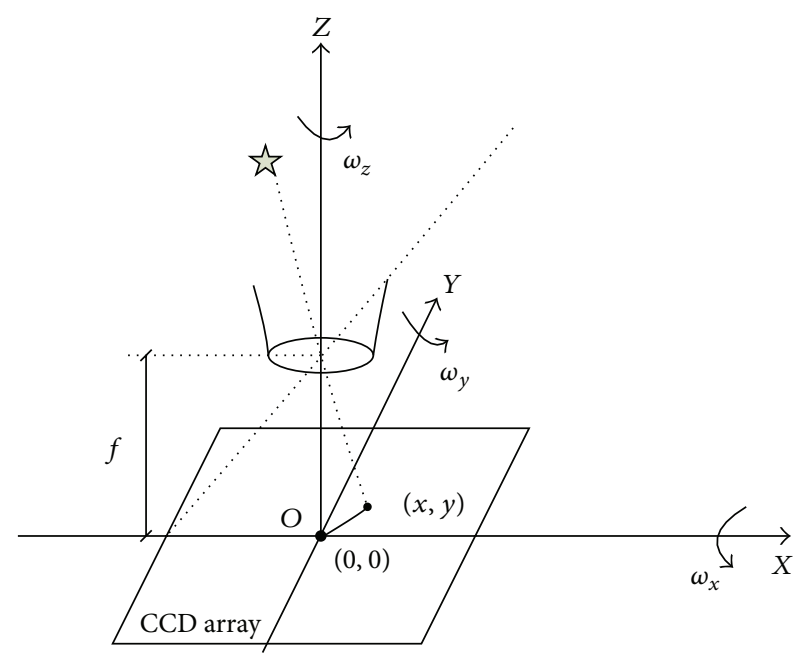

FiguRE 1: Imaging geometrical model of star sensor.

Continuing to evaluate the second-order derivative of (2), a differential equation set can be derived:

$$
\begin{gathered}
x^{\prime \prime}(t)=-y^{\prime}(t) \omega_{z}, \\
y^{\prime}(t)=-f \omega_{x}+x(t) \omega_{z} .
\end{gathered}
$$

Then, a differential equation about $x$ can be obtained:

$$
x^{\prime \prime}(t)=\left(f \omega_{x}-x(t) \omega_{z}\right) \omega_{z} .
$$

Solve (5) and function $x$ can be expressed as

$$
x(t)=c_{1} \cos \left(\omega_{z} t\right)+c_{1} \sin \left(\omega_{z} t\right)+f \frac{\omega_{x}}{\omega_{z}} .
$$

Similarly, function $y$ can be expressed as

$$
y(t)=c_{3} \cos \left(\omega_{z} t\right)+c_{4} \sin \left(\omega_{z} t\right)+f \frac{\omega_{y}}{\omega_{z}} .
$$

$c_{1}, c_{2}, c_{3}$, and $c_{4}$ are constant coefficients of real numbers relying on initial conditions of the smeared star, when $t=0$, $x(0)=x_{0}, y(0)=y_{0}, x^{\prime}(0)=f \omega_{y}-y_{0} \omega_{z}$, and $y^{\prime}(0)=$ $-f \omega_{x}+x_{0} \omega_{z}$. Those constant coefficients can be deduced when the initial conditions are combined with (6) and (7):

$$
\begin{gathered}
c_{1}=c_{4}=x_{0}-f \frac{\omega_{x}}{\omega_{z}}, \\
c_{2}=-c_{3}=f \frac{\omega_{y}}{\omega_{z}}-y_{0} .
\end{gathered}
$$

\section{Characteristics of Smearing Star}

Trajectory of a smearing star has already been deduced above. Gray value of the smeared star in coordinate $(i, j)$ can be calculated in theory [8]:

$$
G_{i j}=\frac{1}{t_{e}} \int_{0}^{t_{e}} g_{i j}(x, y) d t,
$$


where $g_{i j}(x, y)=\left(A_{0} / 2 \pi \sigma^{2}\right) \exp \left(-\left(\left(x_{i}-x(t)\right)^{2}+\right.\right.$ $\left.\left(y_{j}-y(t)\right)^{2}\right) / 2 \sigma^{2}$ ) and $t_{e}$ is the integration time. $x(t)$ and $y(t)$ are components of the trajectory on $X$ axis and $Y$ axis, respectively.

When parameter $t$ is removed the smearing trajectory can be expressed only by parameters $x$ and $y$ :

$$
\left(x-f \frac{\omega_{x}}{\omega_{z}}\right)^{2}+\left(y-f \frac{\omega_{y}}{\omega_{z}}\right)^{2}=c_{1}^{2}+c_{2}^{2} .
$$

The trajectory is along a certain circle, its centre locates at $\left(f\left(w_{x} / w_{z}\right), f\left(w_{y} / w z\right)\right)$ and its radius is of $\sqrt{c_{1}^{2}+c_{2}^{2}}$.

Centre of a star almost keeps motionless on the focal plane during the integration time that is the case in static condition. However, its centre moves along the trajectory in dynamic condition which makes the star a trail imaged on the focal plane.

In reality, a star sensor does not output the pose during the two phases of propelled flight and entry because of the body vibration conducted from the engine and high speed spin of the spacecraft; its only functioning period is the coast phase. In this period, angular rates of the spacecraft can be regarded as constants during its integration time, which is as short as $200 \mathrm{~ms}, 100 \mathrm{~ms}$, or less. This hypothesis is strictly valid for the case of midcourse of a missile in space, when the engine shuts down and no control moment is exerted.

Assuming that the initial condition of a given smeared star is known, its smearing trajectory can be expanded as the first-order Taylor series considering that $t_{e}$ is only a small amount:

$$
\begin{gathered}
x(t)=x_{0}+\left(f \omega_{y}-y_{0} \omega_{z}\right) t+o(t), \\
y(t)=y_{0}+\left(-f \omega_{x}+x_{0} \omega_{z}\right) t+o(t) .
\end{gathered}
$$

When the high-order terms are omitted, the trajectory can be approximated from engineering perspective:

$$
\begin{aligned}
& \widehat{x(t)}=x_{0}+\left(f \omega_{y}-y_{0} \omega_{z}\right) t, \\
& \widehat{y(t)}=y_{0}+\left(-f \omega_{x}+x_{0} \omega_{z}\right) t .
\end{aligned}
$$

This polynomial approximation plays a vital role in the following discussion and will not bring about notable errors when one of the main silicon array detectors is applied. In Section 7.1 the accuracy of this approximation will be analyzed through numerical simulation method.

\section{Errors of Centroid by CoM}

Prior to the computation of centroid, pixels of smeared stars are extracted crudely from the noisy background. Then, size of centroid window for each detected star is determined and the centroid can be calculated by

$$
\begin{aligned}
& x_{c}=\frac{\sum_{i, j}^{L, M} x_{i} I_{i, j}}{\sum_{i, j}^{L . M} I_{i, j}}, \\
& y_{c}=\frac{\sum_{i, j}^{L, M} y_{j} I_{i, j}}{\sum_{i, j}^{L, M} I_{i, j}} .
\end{aligned}
$$

$L \times M$ denotes the size of the centroid window, $i=1 \sim L$, $j=1 \sim M . x_{i}$ and $y_{j}$ represent coordinate of gray on $X$ axis and $Y$ axis, respectively. $I_{i, j}$ denotes the gray value in $(i, j)$ and $\left(x_{c}, y_{c}\right)$ is the mass centre of the star calculated by CoM [3].

$\left(x_{0}, y_{0}\right)$ is the actual position of the given smeared star; it is the position where the star located at the beginning of the integration time and where the star should be in static condition. Relationship between $\left(x_{0}, y_{0}\right)$ and $\left(x_{c}, y_{c}\right)$ can be built when the errors resulting from image smearing on $X$ axis and $Y$ axis are noted as $\Delta x$ and $\Delta y$, respectively:

$$
\begin{aligned}
& x_{0}=x_{c}+\Delta x, \\
& y_{0}=y_{c}+\Delta y .
\end{aligned}
$$

Movement of the centre of smearing star can be served as uniform motion in straight line approximately during the short integration time. $\left(x_{c}, y_{c}\right)$ can be taken as the location where centre of the star is when time $t$ is exactly at half of the integration time [7]. Figure 2 shows the position relation instructed above. Namely,

$$
\begin{array}{r}
x_{c}=x\left(\frac{1}{2} t_{e}\right)=x_{0}+\frac{1}{2}\left(f \omega_{y}-y_{0} \omega_{z}\right) t_{e}+o(t), \\
0 \leq t \leq t_{e}, \\
y_{c}=y\left(\frac{1}{2} t_{e}\right)=y_{0}+\frac{1}{2}\left(-f \omega_{x}+x_{0} \omega_{z}\right) t_{e}+o(t), \\
0 \leq t \leq t_{e} .
\end{array}
$$

$\left(x_{0}, y_{0}\right)$ is substituted by $\left(x_{c}+\Delta x, y_{c}+\Delta y\right)$ in (15) and relationship between $\left(x_{c}, y_{c}\right)$ and $(\Delta x, \Delta y)$ can be built:

$$
\begin{aligned}
\Delta x+\frac{1}{2} t_{e} \omega_{z} \Delta y & =\frac{1}{2} t_{e} f \omega_{y}-\frac{1}{2} t_{e} y_{c} \omega_{z}+o(t), \\
-\frac{1}{2} t_{e} \omega_{z} \Delta x+\Delta y & =-\frac{1}{2} t_{e} f \omega_{x}-\frac{1}{2} t_{e} x_{c} \omega_{z}+o(t) .
\end{aligned}
$$

$\left|\begin{array}{cc}1 & (1 / 2) t_{e} \omega_{z} \\ -(1 / 2) t_{e} \omega_{z} & 1\end{array}\right|>0$ is set up constantly and equation set (16) has the unique solution

$$
\begin{aligned}
\Delta x= & \left(\frac{1}{2} t_{e} f \omega_{y}-\frac{1}{2} t_{e} y_{c} \omega_{z}\right. \\
& \left.+\frac{1}{4} t_{e}^{2} f \omega_{x} \omega_{z}-\frac{1}{4} t_{e}^{2} \omega_{z}^{2} x_{c}\right) \\
& \times\left(1+\frac{1}{4} t_{e}^{2} \omega_{z}^{2}\right)^{-1}+\mathrm{o}(t), \\
\Delta y= & \left(-\frac{1}{2} t_{e} f \omega_{x}+\frac{1}{2} t_{e} x_{c} \omega_{z}\right. \\
& \left.+\frac{1}{4} t_{e}^{2} f \omega_{y} \omega_{z}-\frac{1}{4} t_{e}^{2} \omega_{z}^{2} y_{c}\right) \\
& \times\left(1+\frac{1}{4} t_{e}^{2} \omega_{z}^{2}\right)^{-1}+\mathrm{o}(t) .
\end{aligned}
$$

Analytical expressions of the errors caused by image smearing for centroid estimation are deduced withhigh-order term o $(t)$ (17). Four things become apparent from the results: (1) the variation of $t_{e}$ and $f$ can affect the degree of the smear; (2) a detector with suitable pixel size should be chosen to increase the performance of the star sensor in dynamic 
condition; (3) position of a star in the FOV is also a weighty element, usually ignored, and might also affect its smearing degree; (4) angular rate of the spacecraft might vary all the time during the flight and also contribute to the smearing of the star image as would be discussed later.

In the following section, a novel centroid method developed from the basic CoM will be proposed. It might process better centroid when star sensor works in high dynamic condition.

\section{Processing Centroid}

$t_{e}$ is a small amount and $0 \leq t \leq t_{e}$ is set up all the time. The high-order terms (in (17)) might be omitted from engineering perspective. Then the errors resulting from image smearing might be estimated:

$$
\begin{aligned}
& \widehat{\Delta x}=( \frac{1}{2} t_{e} f \omega_{y}-\frac{1}{2} t_{e} y_{c} \omega_{z} \\
&\left.+\frac{1}{4} t_{e}^{2} f \omega_{x} \omega_{z}-\frac{1}{4} t_{e}^{2} \omega_{z}^{2} x_{c}\right) \times\left(1+\frac{1}{4} t_{e}^{2} \omega_{z}^{2}\right)^{-1} \\
& \widehat{\Delta y}=\left(-\frac{1}{2} t_{e} f \omega_{x}+\frac{1}{2} t_{e} x_{c} \omega_{z}\right. \\
&\left.\quad+\frac{1}{4} t_{e}^{2} f \omega_{y} \omega_{z}-\frac{1}{4} t_{e}^{2} \omega_{z}^{2} y_{c}\right) \times\left(1+\frac{1}{4} t_{e}^{2} \omega_{z}^{2}\right)^{-1}
\end{aligned}
$$

$\widehat{\Delta x}$ and $\widehat{\Delta y}$ denote the estimated values of $\Delta x$ and $\Delta y$, respectively.

Although $\Delta x$ and $\Delta y$ cannot be ignored, they both can be estimated with high accuracy. In (18), $t_{e}$ and $\left(x_{c}, y_{c}\right)$ are sure for star sensor in real time. Usually, a star sensor has been calibrated on ground before it is launched with a spacecraft, so $f$ is sure in advance. Even though $f$ may drift away from the calibrated value, it can be estimated with high precision [9]. In addition, the angular rates are available by the embedded gyroscope or can be estimated by Kalman filtering [10-12].

A flowchart of the proposed centroid-estimation algorithm is shown in Figure 3. The subpixel centroid is calculated in the following way. Grays of the stars are extracted from star image firstly. At the same time, the number of stars detected is counted. Star tracker will not output the pose of the spacecraft when the number of detected stars is less than 3. Num_star denotes the number of stars detected in the FOV. Afterwards, centre of each star is estimated by CoM. Then $\left(x_{c}, y_{c}\right)$ is used to estimate the error caused by image smearing for each detected star. Lastly, $\left(x_{c}, y_{c}\right)$ is revised by $(\widehat{\Delta x}, \widehat{\Delta y})$.

\section{Results and Analysis}

Digital values of light intensity for a smeared star can be calculated (9). Firstly, a mimic image of gray values resulting from the rotation of the spacecraft is obtained for a star. Afterwards, all the other stars in the FOV are simulated. Then, a smeared star image can be formed and all the smeared stars in it have an exact position. Furthermore, angular rates and $t_{e}$ are changeable and star images with different smearing degree can be simulated which might help to validate the efficiency of the proposed method.

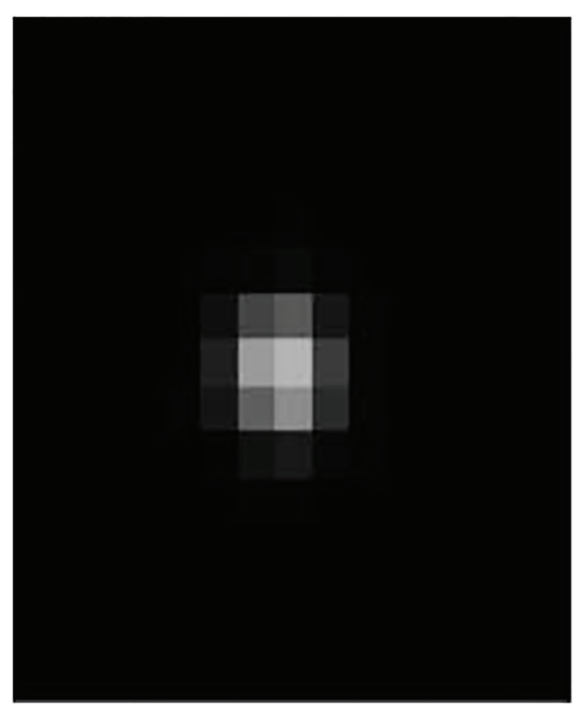

(a)

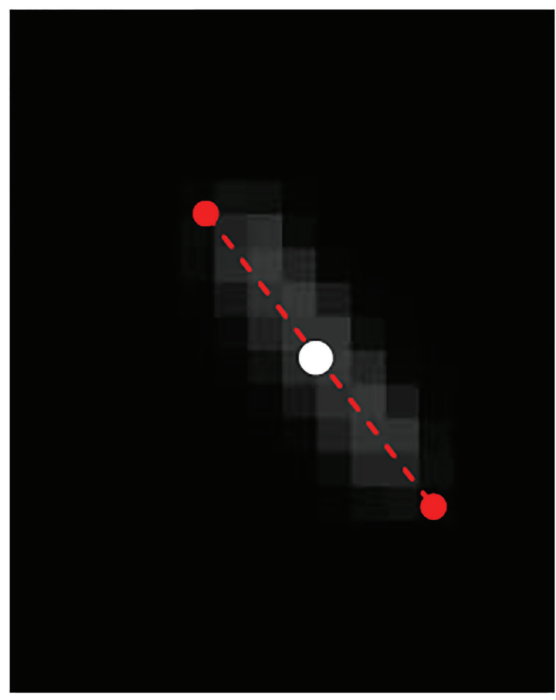

(b)

Figure 2: (a) A static star spot cropped from star image (b) movement of the centre of a smearing star.

All the simulations and experiments discussed herein are based on MATLAB 2010 in PC. Several parameters are set constantly. $f$ is set to $60 \mathrm{~mm}$; spread of the PSF is 0.6 of a pixel, ensuring that more than $95 \%$ energy of the star is restricted within a 3 by 3 pixels area when it is imaged in static condition and pixel size is 15 by $15 \mu \mathrm{m}^{2}$.

7.1. Precision Analysis of Polynomial Approximation. Trajectory of a smearing star can be deduced when $\omega_{z}$ is zero in the same way as it was in Section 3. Namely,

$$
\begin{gathered}
x(t)=f \omega_{y} t+x_{0}, \\
y(t)=-f \omega_{x} t+y_{0} .
\end{gathered}
$$

It shows that $\omega_{z}$ makes the trajectory a small section of an arc while $\omega_{x}$ and $\omega_{y}$ can only drive the star moving along a straight line strictly. As proves that $\omega_{z}$ is the factor 


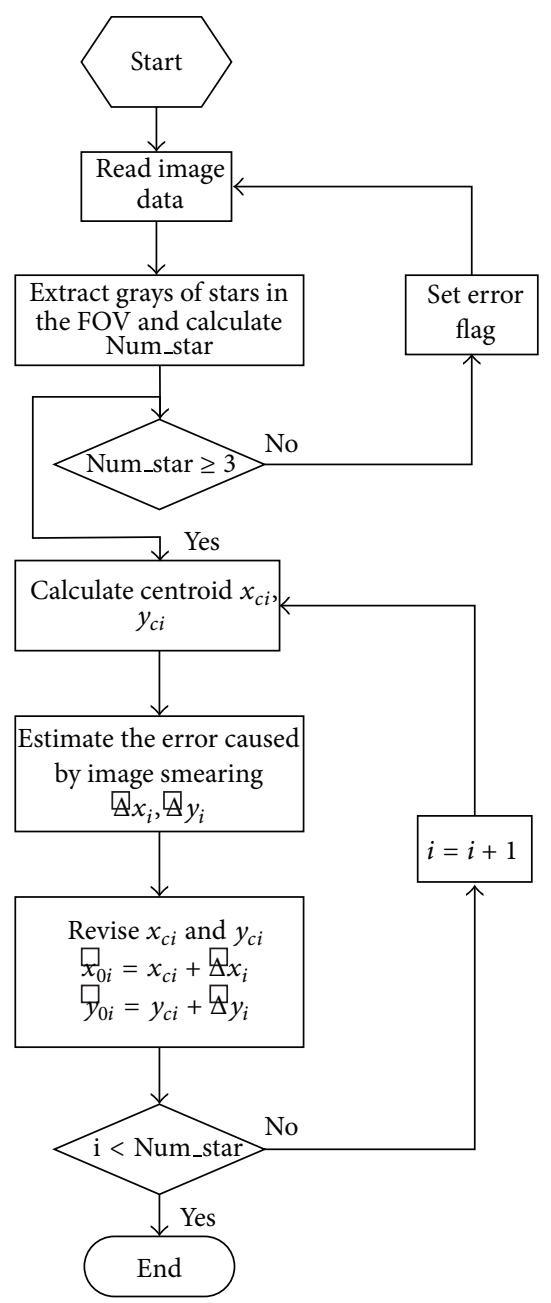

FIGURE 3: Flowchart of the proposed centroid-estimation algorithm.

which contributes to the errors resulting from the polynomial approximation (12).

Maximum value of the difference between analytical form of the trajectory and its approximated form (20) might depict the errors caused by the overlook of the high-order term. Namely,

$$
\begin{aligned}
& x_{\text {error }}=\operatorname{Max}(|x(t)-\widehat{x(t)}|), \\
& y_{\text {error }}=\operatorname{Max}(|y(t)-\widehat{y(t)}|),
\end{aligned}
$$

where $x_{\text {error }}$ and $y_{\text {error }}$ represent the error caused by the overlook of the high-order terms.

A simulation was run. $t_{e}$ was set to $100 \mathrm{~ms} . \omega_{x}$ and $\omega_{y}$ both kept $3.0 \mathrm{deg} / \mathrm{s} . \omega_{z}$ was the only factor changeable, varying from $1.0 \mathrm{deg} / \mathrm{s}$ to $20.0 \mathrm{deg} / \mathrm{s}$. The smeared star located at $(150.0000,150.0000)$, which might help to eliminate the effect caused by varied position of the star.

Both $x_{\text {error }}$ and $y_{\text {error }}$ increased with the increase of $\omega_{z}$ and reached their peaks when $\omega_{z}$ increased to $20.0 \mathrm{deg} / \mathrm{s}$ (Figure 4). Actually, $\omega_{z}$ is far lower than $20.0 \mathrm{deg} / \mathrm{s}$ during functioning period of the star sensor. However, the peak was still lower than $1 / 20$ of a pixel for such an incredible range, demonstrating that the polynomial approximations

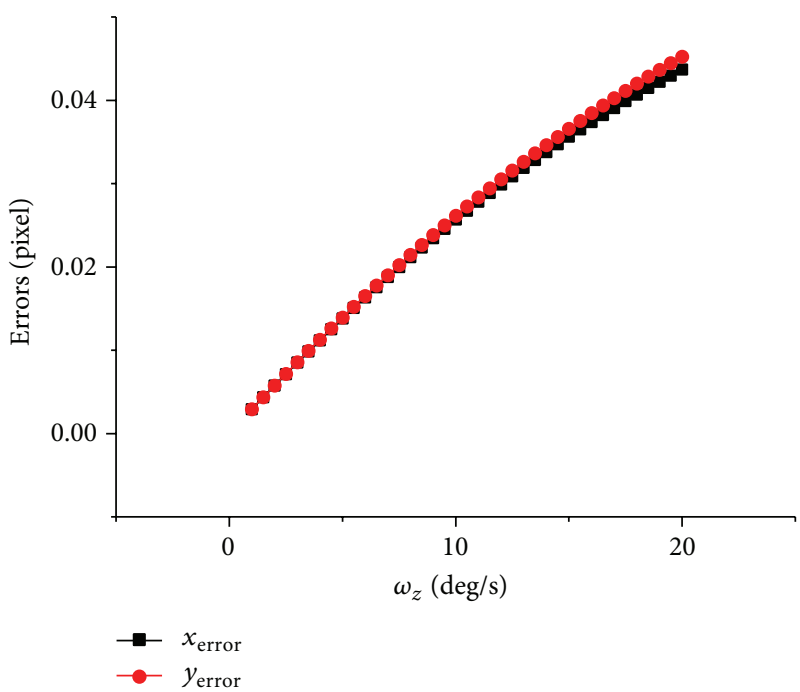

FIGURE 4: Error of the approximation of $x(t)$ and $y(t)$.

of the smearing trajectory are suitable from engineering perspective.

7.2. Effects of Vital Elements on Processing Centroid. Smearing degree of an imaged star in dynamic condition is determined by many parameters. Actual position and integration time are important elements. In this section we will further study how those elements have effect on the accuracy of processed centroid.

Stars in different positions of the FOV will smear with difference. A simulation was constructed to study the relationship. All the angular rates kept $3.0 \mathrm{deg} / \mathrm{s} .\left(x_{0}, y_{0}\right)$ was set to $(50.0000,50.0000),(100.0000,100.0000),(150.0000$, $150.0000),(200.0000,200.0000),(250.0000,250.0000)$, and $(300.0000,300.0000)$ successively, meaning that the distance between the simulated star and $Z$ axis (Figure 1 ) became longer. CoM was used to process centroid and the calculated $\Delta x$ and $\Delta y$ were viewed in Figure 5.

The results indicate that the farther a star is away from $Z$ axis the more serious the smearing of star will be. $\Delta x$ and $\Delta y$ alike reach about 4.5 pixels when the actual position is set at $(300.0000,300.0000)$. Hence, stars near the centre of the focal plane will have a better accuracy when CoM is applied.

Another simulation was run to study the accuracy when $t_{e}$ varied. It varied from $20 \mathrm{~ms}$ to $180 \mathrm{~ms}$ which met the range of the integration time that a star sensor spent to output the star image. Actual position was set at $(150.0000,150.0000)$ and all the angular rates were $1.0 \mathrm{deg} / \mathrm{s}$.

Results of this simulation analysis are shown in Figure 6. The variation of $\Delta x$ and $\Delta y$ indicates that the bias errors, the absolute values of $\Delta x$ and $\Delta y$, increase when $t_{e}$ becomes longer. For a certain star, the smearing length increases and gray intensity grows thinner. That is to say, energy of stars has the probability of being swallowed by the noisy background. Crude extraction of stars from the background will be a headscratching problem. Clearly, $t_{e}$ should be suitable, ensuring the efficient accumulation of star energy by detector and decimating the effect of the noisy background on estimating centroid simultaneity. 


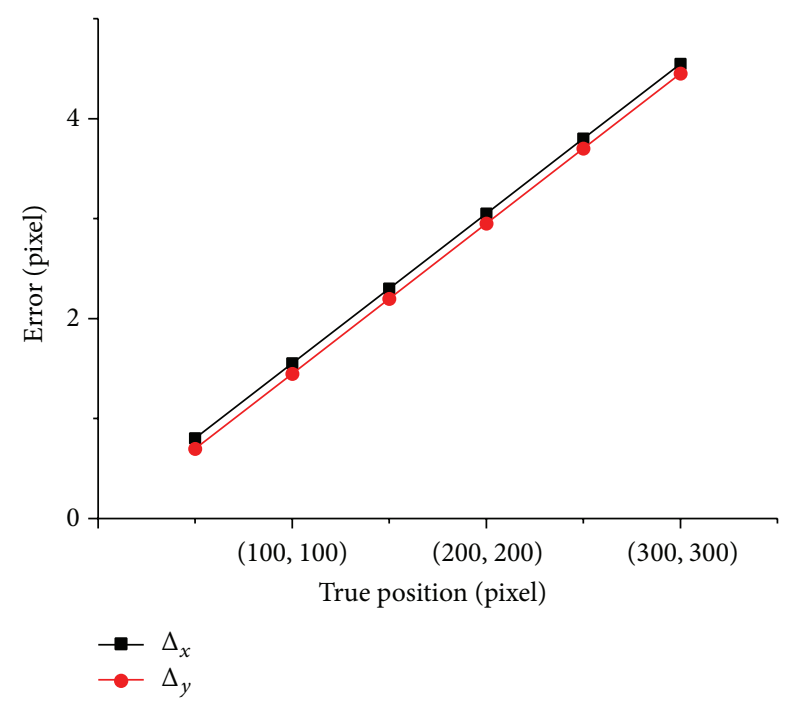

FIGURE 5: Errors vary with the actual position.

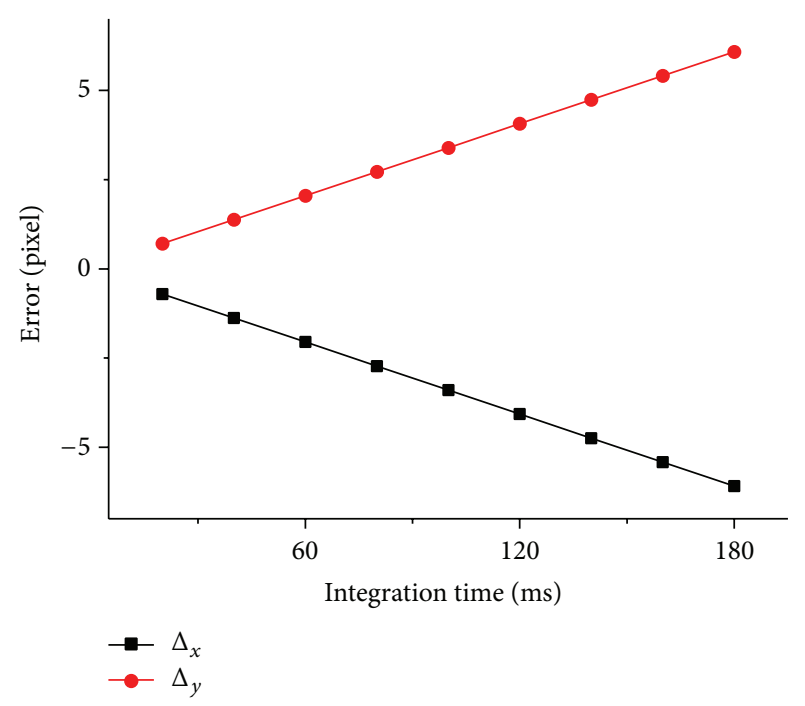

FIgURE 6: Errors vary with the integration time $\left(t_{e}\right)$.

7.3. Estimate Centroid. The bias errors may reach several pixels when CoM is applied to a smeared star. A star sensor can hardly output the pose of the carrier in that situation. In this section, our method would be applied to smeared stars. Comparison between our method and CoM will be drawn to verify the feasibility of our method.

Case 1. Actual positions of smeared stars all were (150.0000, $150.0000) ; \omega_{x}$ increased from $0.5 \mathrm{deg} / \mathrm{s}$ to $3.0 \mathrm{deg} / \mathrm{s}$ while $\omega_{y}$ and $\omega_{z} \mathrm{kept} 1.0 \mathrm{deg} / \mathrm{s}$. In the presence of white Gaussian noise, the SNR (signal to noise ratio) was $60 \mathrm{~dB}$ indicating a low noise level.

$\Delta x, \Delta y, \widehat{\Delta x}, \widehat{\Delta y}$, and the estimated centroid are listed in Table 1. With the increase of $\omega_{x}, \Delta x$ keeps about -3.4 pixels while $\Delta y$ increases and reaches about 10.5 pixels when $\omega_{x}$ increases to $3.0 \mathrm{deg} / \mathrm{s}$. However, our method has the accuracy better than $1 / 10$ of a pixel even when $\omega_{x}$ increases to $3.0 \mathrm{deg} / \mathrm{s}$ which is the peak set in the simulation. Meanwhile, we can see that $\Delta y$ increases while $\Delta x$ keeps almost constant with the increase of $\omega_{x}$. The variation of $\omega_{x}$ might cause the variation of $\Delta y$ while it might not be attributed to the variation of $\Delta x$, just as it was described in (3).

Case 2. $\left(x_{0}, y_{0}\right)$ of smeared stars were set to (150.0000, $150.0000) ; \omega_{y}$ increased from $0.5 \mathrm{deg} / \mathrm{s}$ to $3.0 \mathrm{deg} / \mathrm{s}$ while $\omega_{x}$ and $\omega_{z}$ were $1.0 \mathrm{deg} / \mathrm{s}$ constantly. The noise level was $60 \mathrm{~dB}$.

When $\omega_{y}$ increases from $1.0 \mathrm{deg} / \mathrm{s}$ to $3.0 \mathrm{deg} / \mathrm{s} \Delta y$ keeps about 3.4 pixels. But $\Delta x$ decreases from -3.4 pixels to -10.4 pixels, meaning that bias error in $X$ axis increases (Table 2). What also draws our attention is that the variation of $\omega_{y}$ might cause the variation of $\Delta x$ while it might not be attributed to the variation of $\Delta y(2)$. Our method shows that the results are better than $1 / 10$ of a pixel even when $\omega_{y}$ reaches about $3.0 \mathrm{deg} / \mathrm{s}$.

Case 3. Actual positions are all set to $(150.0000,150.0000)$; $\omega_{z}$ increases from $0.5 \mathrm{deg} / \mathrm{s}$ to $3.0 \mathrm{deg} / \mathrm{s}$ while the other two angular rates are $1.0 \mathrm{deg} / \mathrm{s}$ constantly. SNR was $60 \mathrm{~dB}$.

The increase of $\omega_{z}$ has the least influence on the precision of estimation centroid (Table 3 ), because the focal length and the CCD array size are not of the same order of the magnitude, with the former one much higher than the latter one. When the three angular rates are at the same level $\omega_{z}$ contributes the least to the alteration of the line velocities in $X$ axis and $Y$ axis directions ((2) and (3)).

In summary, smearing of star makes the obtained centroid worse than it should be in static condition. Precision of CoM for smeared star is even worse than 1.0 pixel, which will certainly result in the failure of pattern recognition of star image. However, our method provides a concise and efficient way to process the centroid. Revising the centroid, processed by CoM, with the corresponding errors estimated by (18), accuracy of the processed centroid can be better than 1/10 of a pixel for smearing stars.

\section{Conclusion}

The smearing trajectory of star on the focal plane has been modeled. Within the allowed precision, the smearing trajectory has been approximated by polynomial from engineering prospective. Effects of actual position of star, integration time, pixel size, focal length, and angular rates on the accuracy of subpixel centroid estimation have been modeled in analytical form.

Also, simulations have been run to further study the effects of integration time, actual position, and angular rates on the accuracy of processed centroid. When the integration time becomes longer the length of smearing also gets longer. It is suggested that the estimated centroids of stars which are near the centre of the focal plane are proved to be less affected by the rotation of the star sensor around $Z$ axis. Also, it is proved that among the three angular rates $\omega_{z}$ has the least effect on the bias errors; $\omega_{x}$ can only contribute to $\Delta y$ and $\omega_{y}$ can only contribute to $\Delta x$. 
TABLE 1: Bias errors and the revised centroid with the increase of $\omega_{x}$.

\begin{tabular}{|c|c|c|c|c|c|c|c|}
\hline$\omega_{x}(\mathrm{deg} / \mathrm{s})$ & $\begin{array}{l}\text { Angular rates } \\
\omega_{y}(\mathrm{deg} / \mathrm{s})\end{array}$ & $\omega_{z}(\mathrm{deg} / \mathrm{s})$ & $\Delta x($ pixel $)$ & $\Delta y($ pixel $)$ & $\widehat{\Delta x}($ pixel $)$ & $\widehat{\Delta y}($ pixel $)$ & $\left(\widehat{x_{0}}, \widehat{y_{0}}\right)($ pixel, pixel $)$ \\
\hline 1.0 & 1.0 & 1.0 & -3.3953 & 3.3914 & 3.3565 & -3.3565 & $(149.9612,150.0349)$ \\
\hline 1.5 & 1.0 & 1.0 & -3.3964 & 5.1542 & 3.3550 & -5.1017 & $(149.9586,150.0525)$ \\
\hline 2.0 & 1.0 & 1.0 & -3.3974 & 6.9169 & 3.3535 & -6.8468 & $(149.9561,150.0701)$ \\
\hline 2.5 & 1.0 & 1.0 & -3.3984 & 8.6797 & 3.3519 & -8.5920 & $(149.9535,150.0877)$ \\
\hline 3.0 & 1.0 & 1.0 & -3.3995 & 10.4425 & 3.3504 & -10.3371 & $(149.9509,150.1054)$ \\
\hline
\end{tabular}

TABLE 2: Bias errors and the revised centroid with the increase of $\omega_{y}$.

\begin{tabular}{|c|c|c|c|c|c|c|c|}
\hline$\omega_{x}(\mathrm{deg} / \mathrm{s})$ & $\begin{array}{l}\text { Angular rates } \\
\omega_{y}(\mathrm{deg} / \mathrm{s})\end{array}$ & $\omega_{z}(\mathrm{deg} / \mathrm{s})$ & $\Delta x($ pixel $)$ & $\Delta y($ pixel $)$ & $\widehat{\Delta x}($ pixel $)$ & $\widehat{\Delta y}($ pixel $)$ & $\left(\widehat{x_{0}}, \widehat{y_{0}}\right)($ pixel, pixel $)$ \\
\hline 1.0 & 1.0 & 1.0 & -3.3953 & 3.3914 & 3.3565 & -3.3565 & $(149.9612,150.0349)$ \\
\hline 1.0 & 1.5 & 1.0 & -5.1581 & 3.3903 & 5.1017 & -3.3550 & $(149.9435,150.0354)$ \\
\hline 1.0 & 2.0 & 1.0 & -6.9209 & 3.3893 & 6.8468 & -3.3535 & $(149.9259,150.0359)$ \\
\hline 1.0 & 2.5 & 1.0 & -8.6837 & 3.3883 & 8.5920 & -3.3519 & $(149.9083,150.0364)$ \\
\hline 1.0 & 3.0 & 1.0 & -10.4465 & 3.3872 & 10.3371 & -3.3504 & $(149.8907,150.0368)$ \\
\hline
\end{tabular}

TABLE 3: Bias errors and the revised centroid with the increase of $\omega_{z}$.

\begin{tabular}{|c|c|c|c|c|c|c|c|}
\hline$\omega_{x}(\operatorname{deg} / \mathrm{s})$ & $\begin{array}{c}\text { Angular rates } \\
\omega_{y}(\mathrm{deg} / \mathrm{s})\end{array}$ & $\omega_{z}(\mathrm{deg} / \mathrm{s})$ & $\Delta x($ pixel $)$ & $\Delta y($ pixel $)$ & $\widehat{\Delta x}($ pixel $)$ & $\widehat{\Delta y}($ pixel $)$ & $\left(\widehat{x_{0}}, \widehat{y_{0}}\right)($ pixel, pixel $)$ \\
\hline 1.0 & 1.0 & 1.0 & -3.3953 & 3.3914 & 3.3565 & -3.3565 & $(149.9612,150.0349)$ \\
\hline 1.0 & 1.0 & 1.5 & -3.3302 & 3.3243 & 3.2895 & -3.2895 & $(149.9593,150.0348)$ \\
\hline 1.0 & 1.0 & 2.0 & -3.2650 & 3.2573 & 3.2226 & -3.2226 & $(149.9576,150.0347)$ \\
\hline 1.0 & 1.0 & 2.5 & -3.1997 & 3.1904 & 3.1557 & -3.1557 & $(149.9560,150.0346)$ \\
\hline 1.0 & 1.0 & 3.0 & -3.1344 & 3.1234 & 3.0889 & -3.0889 & $(149.9545,150.0345)$ \\
\hline
\end{tabular}

By revising the centroid (processed by CoM) with the estimated errors, it can be better than $1 / 10$ of a pixel in high dynamic condition, validated by simulation method. Moreover, our method will be useful in the field where high accuracy of pose measurement is needed, based on point targets, in high dynamic condition, such as adaptive optics, spatial acquisition systems for optical links, and laser triangulation sensor.

\section{Conflict of Interests}

The authors declare that there is no conflict of interests regarding the publication of this paper.

\section{Acknowledgments}

The authors thank Xiangdong Zhou and Wenming Zhang for their technical support. The support for this work was provided by the National Key Basic Research Program of China Grant no. 2014CB744200. Special thanks are to the anonymous referees for their thorough review and useful comments.

\section{References}

[1] C. C. Liebe, "Accuracy performance of star trackersm-dasha tutorial," IEEE Transactions on Aerospace and Electronic Systems, vol. 38, no. 2, pp. 587-599, 2002.
[2] C. C. Liebe, E. W. Dennison, B. Hancock, R. C. Stirbl, and B. Pain, "Active pixel sensor (APS) based star tracker," in Proceedings of the IEEE Aerospace Conference, vol. 1, pp. 119-127, March 1998.

[3] A. M. Nightingale and S. Gordeyev, "Shack-Hartmann wavefront sensor image analysis: a comparison of centroiding methods and image-processing techniques," Optical Engineering, vol. 52, pp. 1-21, 2013.

[4] W. Xiaojuan and W. Xinlong, "Multiple blur of star image and the restoration under dynamic conditions," Acta Astronautica, vol. 68, no. 11-12, pp. 1903-1913, 2011.

[5] Z. Weina, Q. Wei, and G. Lei, "Blurred star image processing for star sensor under dynamic conditions," Sensor, vol. 12, pp. 67126726, 2012.

[6] J. Núnez, O. Fors, and A. Prades, "Using image deconvolution to increase the ability to detect stars and faint orbital objects in CCD imaging," in Proceedings of the International Conference on Digital Image Processing, vol. 12, pp. 195-199, March 2009.

[7] M. A. Samaan, T. C. Pollock, and J. L. Junkins, "Predictive centroiding for star trackers with the effect of image Smear," Journal of the Astronautical Sciences, vol. 50, no. 1, pp. 113-123, 2002.

[8] H. Wang, W. Zhou, X. Cheng, and H. Lin, "Image smearing modeling and verification for strapdown star sensor," Chinese Journal of Aeronautics, vol. 25, no. 1, pp. 115-123, 2012.

[9] M. A. Samaan, D. Mortari, and J. L. Junkins, "Autonomous onOrbit calibration of star trackers," in Proceedings of the Core Technologies for Space Systems Conference, Colorado Springs, Colo, USA, November 2001. 
[10] H.-Y. Kim, J.-N. Juang, and J. L. Junkins, "Recursive attitude prediction," Journal of the Astronautical Sciences, vol. 48, no. 2-3, pp. 409-421, 2000.

[11] J. L. Crassidis and F. L. Markley, "Predictive filtering for attitude estimation without rate sensors," Journal of Guidance, Control, and Dynamics, vol. 20, no. 3, pp. 522-527, 1997.

[12] P. Lu, "Nonlinear predictive controllers for continuous systems," Journal of Guidance, Control, and Dynamics, vol. 17, no. 3, pp. 553-560, 1994. 


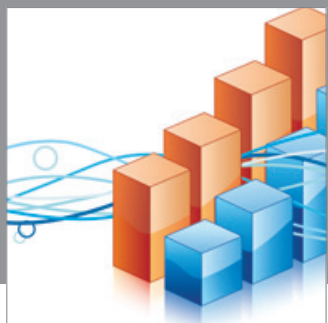

Advances in

Operations Research

mansans

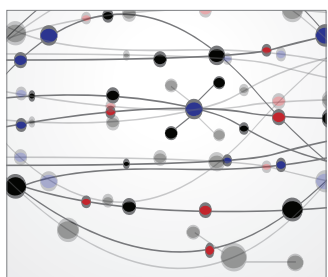

The Scientific World Journal
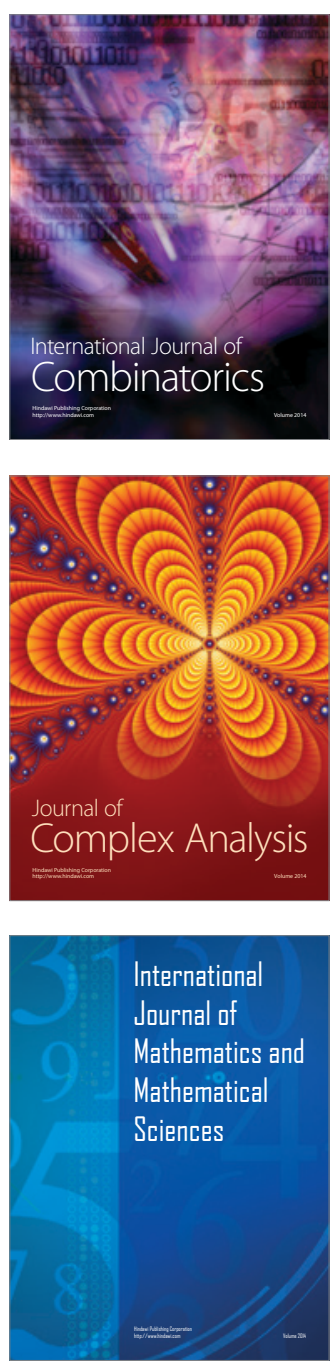
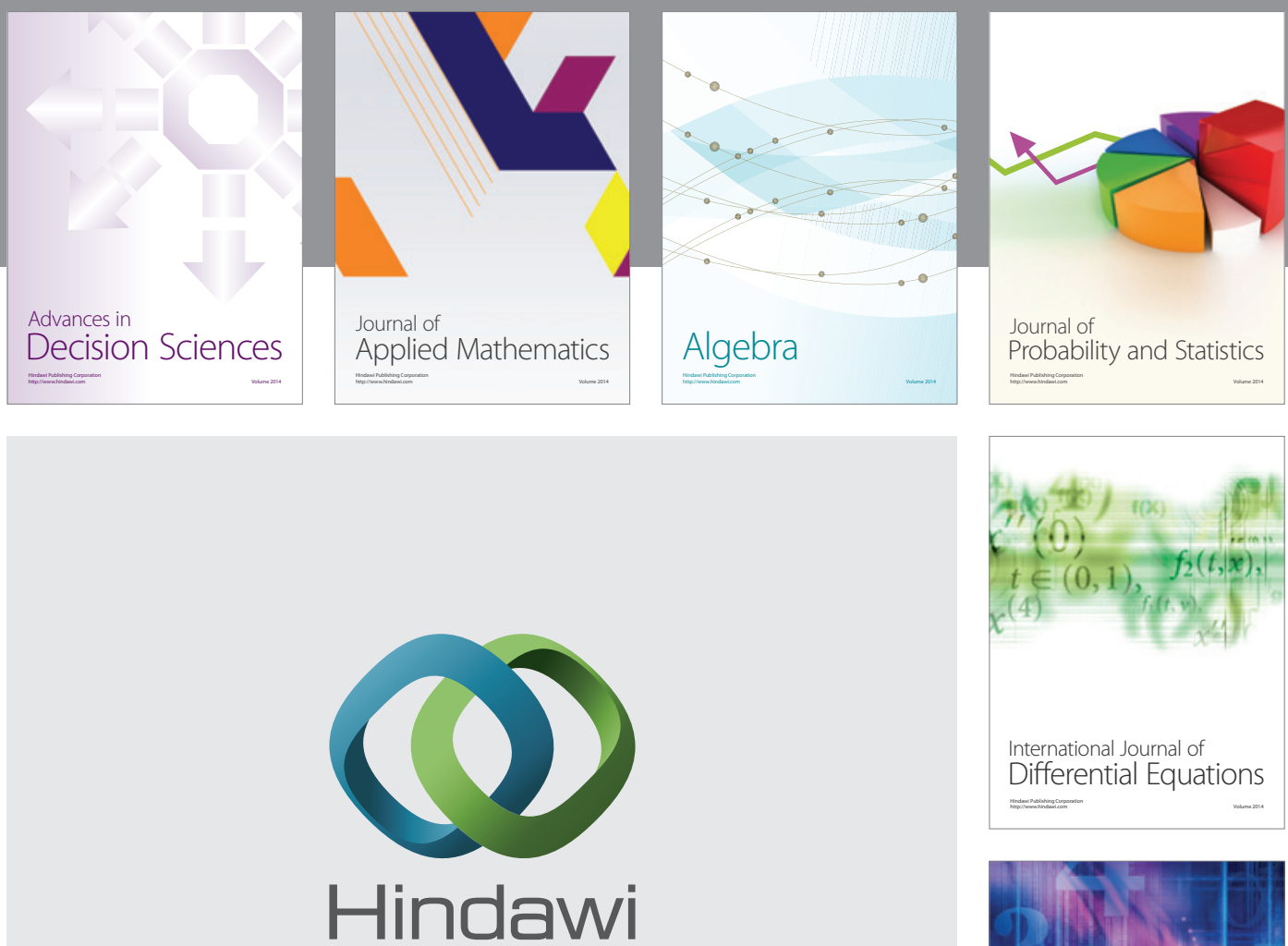

Submit your manuscripts at http://www.hindawi.com
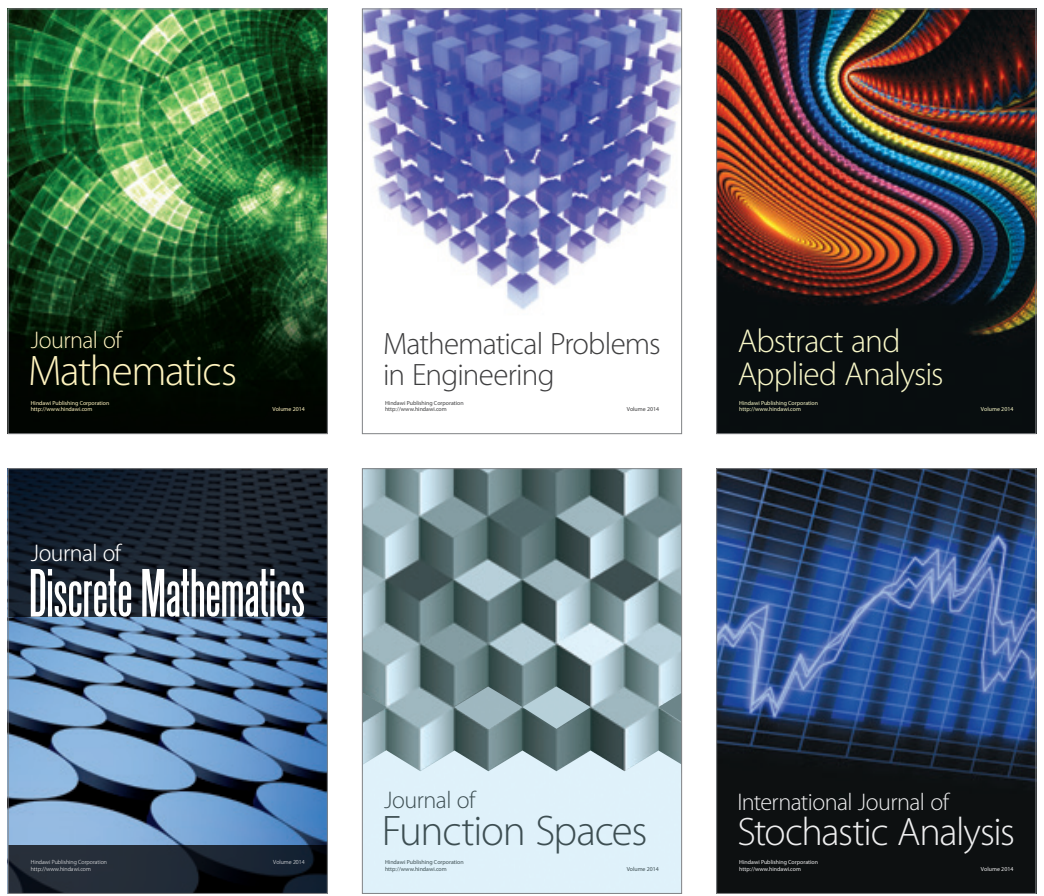

Journal of

Function Spaces

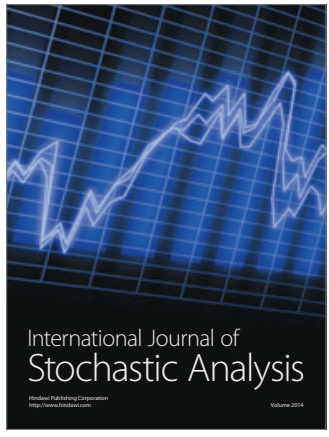

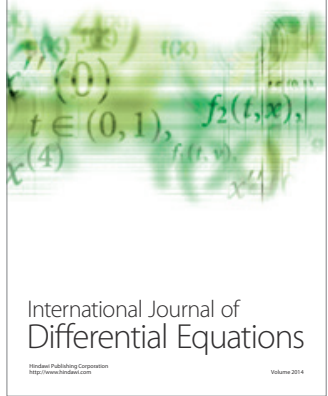
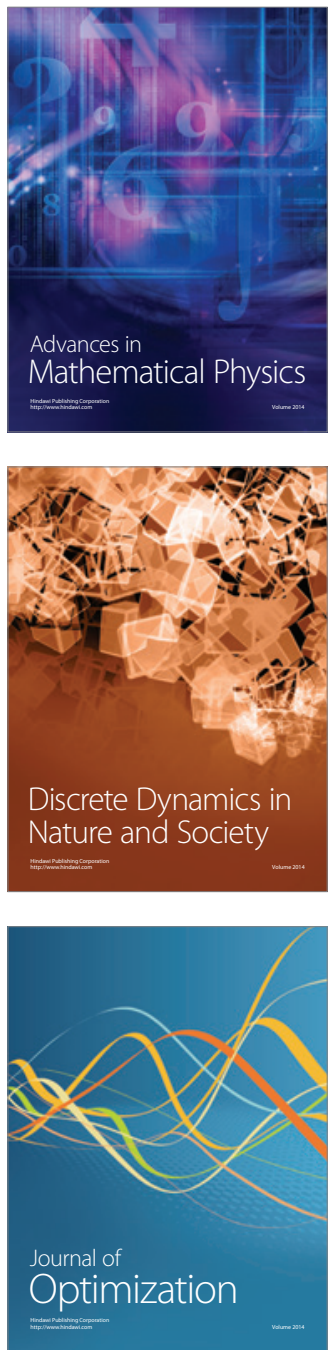